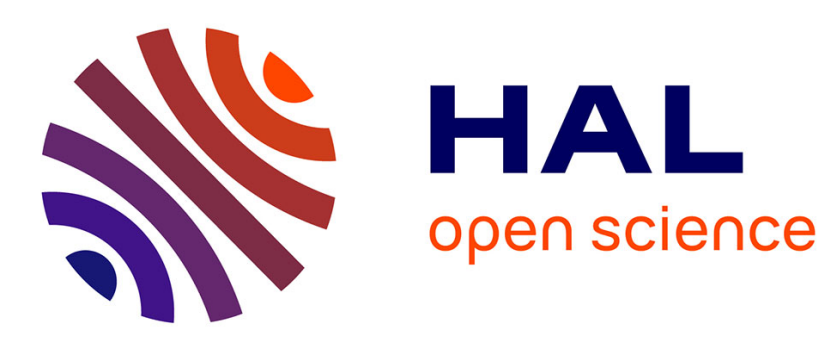

\title{
Multidomain modeling of the influence of plastic deformation on the magnetic behavior
}

\author{
Olivier Hubert, Said Lazreg
}

\section{To cite this version:}

Olivier Hubert, Said Lazreg. Multidomain modeling of the influence of plastic deformation on the magnetic behavior. IEEE Transactions on Magnetics, 2012, 48 (4), pp.1277 - 1280. 10.1109/TMAG.2011.2172935 . hal-01532891

\section{HAL Id: hal-01532891 \\ https://hal.science/hal-01532891}

Submitted on 4 Jun 2017

HAL is a multi-disciplinary open access archive for the deposit and dissemination of scientific research documents, whether they are published or not. The documents may come from teaching and research institutions in France or abroad, or from public or private research centers.
L'archive ouverte pluridisciplinaire HAL, est destinée au dépôt et à la diffusion de documents scientifiques de niveau recherche, publiés ou non, émanant des établissements d'enseignement et de recherche français ou étrangers, des laboratoires publics ou privés. 


\section{Multidomain modeling of the influence of plastic deformation on the magnetic behavior}

\author{
Olivier Hubert \\ LMT-Cachan \\ ENS-Cachan/CNRS/UPMC/UniverSud Paris \\ 61 avenue du président Wilson \\ 94235-F Cachan cedex \\ Email: hubert@lmt.ens-cachan.fr
}

\author{
Said Lazreg \\ LMT-Cachan \\ ENS-Cachan/CNRS/UPMC/UniverSud Paris \\ 61 avenue du président Wilson \\ 94235-F Cachan cedex \\ Email: lazreg@1mt.ens-cachan.fr
}

\begin{abstract}
Magnetic non destructive methods are frequently used in the industries of steel since magnetic behavior demonstrates a good sensitivity to the microstructural and/or mechanical changes. Magnetic behavior is especially sensitive to plastic straining that occurs for example with the manufacturing of materials (cutting, punching). Evaluating the state of a material from a measurement requires a modeling of the behaviors of the material (local constitutive laws) involved that must on the other hand demonstrate low computation time. Such magneto-mechanical modeling is proposed, based on the so-called multidomain model.
\end{abstract}

\section{INTRODUCTION}

The influence of plastic deformation on the magnetic behavior has been studied intensively in the past years. It is characterized by a strong non-linear degradation of the magnetic behavior associated to a shift of the magnetostrictive behavior [1], [2], [3]; it can be interpreted in term of influence of internal stresses and has been modeled thanks to a combination between a micro-macro mechanical modeling of plastic strain and a magneto-elastic multiscale model [4]. This modeling is nevertheless restricted to small plastic strain amplitudes and the calculation time is prohibitive. Application in non destructive evaluation (NDE) area is consequently compromised especially when inverse identification is wanted. A new fast and simple modeling is relevant for that purpose. The modeling proposed herein requires first the calculation of residual stress fields considering the material as a two phased material. The so-called multidomain modeling recently proposed [8] is used for the calculation of the magnetic quantities. The inverse identification procedure is not discussed.

\section{Previous EXPeriments AND MODELING}

It is well known that plastic deformations lead to a sharp degradation of the magnetic properties of ferromagnetic materials (strong decrease of susceptibility, increase of hysteresis losses) [1], [3] especially for weak plastic strain levels. Plastic deformations change on the other hand the magnetostriction amplitude [2], [5]. Classical magnetoelasticity is able to explain these phenomenon assuming that the driving force associated to plasticity is the long range internal stresses [1], [6], [7]. A micro-macro model was previously proposed to describe the influence of plastic strain on the magnetomechanical behavior. It involves first a microcrystalline plasticity approach where the material is defined by its orientation data file (ODF - about 400 grains). The local stress in a grain $\sigma$ is calculated as function of the macroscopic stress $\boldsymbol{\Sigma}$, the local plastic deformation $\boldsymbol{\epsilon}^{p}$ and the macroscopic plastic deformation $\mathbf{E}^{p}$. The effect of plastic deformation at the unloaded state (relaxation of macroscopic stress tensor to zero) after uniaxial traction was modeled.

The next step consists to use the residual stress tensor $\sigma$ as a loading at the grain scale of a magnetic multiscale model able to describe magnetic and magnetostrictive behaviors [4]. This model was applied to non-oriented $\mathrm{Fe}-3 \% \mathrm{Si}$ and simulations of the effect of plasticity are consistent with experimental observations [4], [6]. This approach is complemented by experimental observations where the kinematic hardening (see next section) is correlated with the degradation of the magnetic properties [1]. However, this approach is limited to the range of levels of plastic deformation corresponding to intergranular internal stresses (stress homogeneous at the grain scale). In addition, only monotonic loading is taken into account and only the unloaded state is modeled. This modeling leads on the other hand to dissuasive computation times. Inverse identification is consequently still not reachable by this way. Nevertheless the principle that plasticity can be considered as a state of internal stress is kept for the modeling proposed herein.

\section{Mechanical MOdELING}

The challenge is to simplify the micro-macro approach in order to meet the time constraints inherent with the calculation specifications for NDE. The modeling proposed requires first the calculation of residual stress fields considering the material as a two phased material as initially proposed by Mughrabi [9]. The effect of plastic deformation on the macroscopic magnetic behavior is supposed to correspond to an average effect of the residual stresses on each phase.

\section{A. Composite model}

At the macroscopic scale, we consider a representative volume element (RVE) consisting of two phases: a soft phase $s$ 
and a hard phase $h$, meaning that $s$ phase exhibits a lower yield stress and strengthening than the $h$ phase. $f_{s}$ and $f_{h}$ indicate the volume fraction of $s$ and $h$ phases. The RVE is submitted to an elastoplastic stress tensor $\boldsymbol{\Sigma} . \mathbf{E}^{e}, \mathbf{E}^{p}$ and $\mathbf{E}$ denote the elastic, plastic and total deformation tensors respectively so that :

$$
\mathbf{E}=\mathbf{E}^{e}+\mathbf{E}^{p}=\mathbb{C}^{-1} \boldsymbol{\Sigma}+\mathbf{E}^{p}
$$

where $\mathbb{C}$ indicates the stiffness tensor of the medium supposed isotropic and homogeneous whatever the phase. The same decomposition can be made for each phase:

$$
\begin{gathered}
\boldsymbol{\epsilon}_{h}=\boldsymbol{\epsilon}_{h}^{e}+\boldsymbol{\epsilon}_{h}^{p}=\mathbb{C}^{-1} \boldsymbol{\sigma}_{h}+\boldsymbol{\epsilon}_{h}^{p} \\
\boldsymbol{\epsilon}_{s}=\boldsymbol{\epsilon}_{s}^{e}+\boldsymbol{\epsilon}_{s}^{p}=\mathbb{C}^{-1} \boldsymbol{\sigma}_{s}+\boldsymbol{\epsilon}_{s}^{p}
\end{gathered}
$$

Macroscopic stress and deformation $\boldsymbol{\Sigma}$ and $\mathbf{E}$ are given by:

$$
\begin{gathered}
\boldsymbol{\Sigma}=f_{h} \boldsymbol{\sigma}_{h}+f_{s} \boldsymbol{\sigma}_{s} \\
\mathbf{E}=f_{h} \boldsymbol{\epsilon}_{h}+f_{s} \boldsymbol{\epsilon}_{s}
\end{gathered}
$$

The local stress is given on the other hand by the Hill's relationship [11] so that:

$$
\begin{aligned}
& \boldsymbol{\sigma}_{s}=\mathbf{\Sigma}+\mathbb{C}^{*}\left(\mathbf{E}-\boldsymbol{\epsilon}_{s}\right) \\
& \boldsymbol{\sigma}_{h}=\boldsymbol{\Sigma}+\mathbb{C}^{*}\left(\mathbf{E}-\boldsymbol{\epsilon}_{h}\right)
\end{aligned}
$$

where $\mathbb{C}^{*}$ indicates the Hill's constraint tensor.

Because of isotropic elasticity, the plastic deformation tensors verify:

$$
\mathbf{E}^{p}=f_{s} \boldsymbol{\epsilon}_{s}^{p}+f_{h} \boldsymbol{\epsilon}_{h}^{p}
$$

so that it is possible to define two residual stress tensors $\mathbf{D}_{s}$ and $\mathbf{D}_{h}$ satisfying:

$$
\begin{gathered}
\boldsymbol{\sigma}_{s}=\boldsymbol{\Sigma}+\mathbf{D}_{s} \\
\boldsymbol{\sigma}_{h}=\boldsymbol{\Sigma}+\mathbf{D}_{h} \\
f_{s} \mathbf{D}_{s}+f_{h} \mathbf{D}_{h}=0
\end{gathered}
$$

We observe that: $\mathbf{D}_{h}=-\frac{f_{s}}{f_{h}} \mathbf{D}_{s}$.

\section{B. Correlation with kinematic hardening}

On the other hand the plastic straining of a material is suitably described by the yield function $f$ which can be expressed as function of the macroscopic deviatoric stress tensor $\mathbf{S}^{1}$, yield stress $\Sigma_{y}$, isotropic $R$ and kinematic $\mathbf{X}$ hardening components [12]:

$$
f(\boldsymbol{\Sigma})=\sqrt{\frac{3}{2}(\mathbf{S}-\mathbf{X}):(\mathbf{S}-\mathbf{X})}-\Sigma_{y}-R
$$

assuming that the strengthening is suitably described by a von Mises criterion. $\mathbf{X}$ tensor is a non linear function of the plastic strain tensor $\mathbf{E}^{p}$ (13), related to the position of the yield function $(f=0)$ in the stress space (figure 1) and representative of heterogeneous and multiaxial residual stress field within the material.

$$
\dot{\mathbf{X}}=g\left(\dot{\mathbf{E}}^{p}\right)
$$

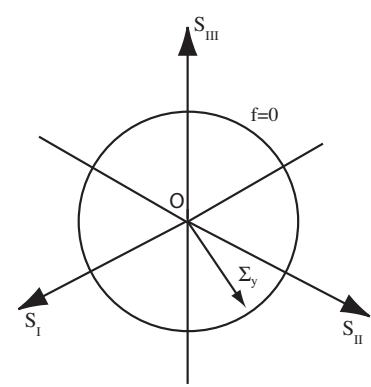

(a)

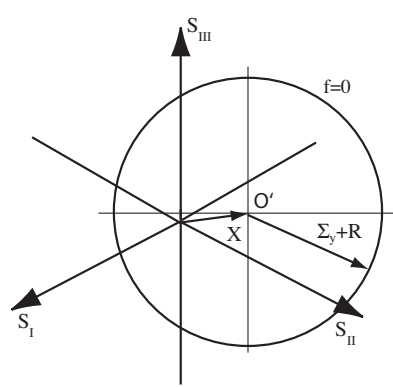

(b)
Fig. 1. Illustration of kinematic and isotropic hardening for a von Mises criterion in the deviatoric eigen-stress space $\left(S_{I}, S_{I I}, S_{I I I}\right)$ : (a) initial yield function; (b) yield function after strengthening.

Coming back to the previous decomposition in soft and hard phases, it can be shown that the kinematic hardening is directly associated to the residual stress within the soft phase, so that:

$$
\mathbf{D}_{s}=-\frac{3}{2} \mathbf{X}
$$

Assuming that the volume fraction of hard and soft phases are known, an experimental estimation of the quantity $\mathbf{X}$ allows to define the stress field within the two phases. Application to tensile strengthening is made in the next section. The procedure is applied experimentally in [13].

\section{Magnetic MOdeling}

\section{A. Multidomain modeling - single phased material [8]}

The multidomain modeling is a two-scales reversible modeling allowing the prediction of the magneto-mechanical behavior of isotropic polycrystals. A six magnetic domains configuration is considered associated to the six easy axes of cubic symmetry for materials that exhibit a positive magnetocrystalline constant (figure 2a). Each domain family $\alpha$ ( $\alpha=1 . .6$ ) is defined by a magnetization vector $\vec{M}_{\alpha}$ (15) so that $\left\|\vec{M}_{\alpha}\right\|=M_{s}$, and by a magnetostriction tensor $\epsilon_{\alpha}^{\mu}(16)$ $\left(\gamma_{i}\right.$ parameters figure the direction cosines of magnetization; $\lambda_{100}$ and $\lambda_{111}$ are the two magnetostrictive constants). This single crystal is considered as submitted to a magnetic field $\vec{H}$ and/or stress $\boldsymbol{\sigma}$. Uniform strain and field hypotheses are used over the crystal and domain walls contribution to the total energy is neglected [10]. The energy of a magnetic domain $W_{\alpha}$ is the sum of the Zeeman energy $W_{\alpha}^{H}$, the magnetocrystalline energy $W_{\alpha}^{K}$ and of the magnetoelastic energy $W_{\alpha}^{\sigma}(17,18,19)$ ( $K_{1}$ is the magnetocrystalline constant of the material). The stress tensor is supposed uniaxial; magnetic field and stress are applied along a same direction $\vec{n}_{c}$ defined by angles $\phi_{c}$ and $\theta_{c}$ of the spherical frame (figure $2 \mathrm{~b}$ ). This direction is restricted to the standard triangle: cubic symmetry means that at any loading direction is corresponding a direction in this triangle. The resolution of the problem (ie calculation of the mean magnetization and deformation) requires to evaluate the direction of magnetization and the volume fraction of each domain family $\alpha$.

\footnotetext{
${ }^{1}$ with $\mathbf{S}=\boldsymbol{\Sigma}-\frac{1}{3} \operatorname{tr}(\boldsymbol{\Sigma}) \mathbf{I} ; \mathbf{I}$ : identity tensor.
} 
(a)

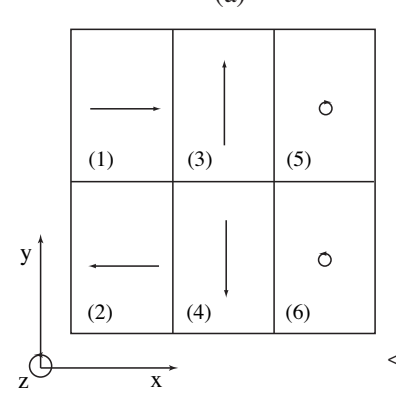

(b)

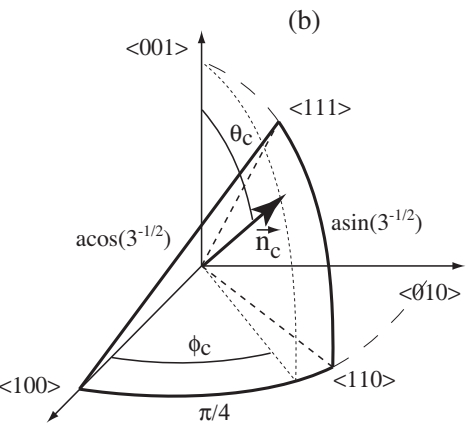

Fig. 2. (a) Initial domain structure of single crystal considered for the multidomain modeling; (b) standard triangle and loading axis $\vec{n}_{c}$.

$$
\begin{aligned}
& \vec{M}_{\alpha}=M_{s}^{t}\left[\gamma_{1}, \gamma_{2}, \gamma_{3}\right] \\
& \boldsymbol{\epsilon}_{\alpha}^{\mu}=\frac{3}{2}\left(\begin{array}{ccc}
\lambda_{100}\left(\left(\gamma_{1}\right)^{2}-\frac{1}{3}\right) & \lambda_{111}\left(\gamma_{1} \gamma_{2}\right) & \lambda_{111}\left(\gamma_{1} \gamma_{3}\right) \\
\lambda_{111}\left(\gamma_{1} \gamma_{2}\right) & \lambda_{100}\left(\left(\gamma_{2}\right)^{2}-\frac{1}{3}\right) & \lambda_{111}\left(\gamma_{2} \gamma_{3}\right) \\
\lambda_{111}\left(\gamma_{1} \gamma_{3}\right) & \lambda_{111}\left(\gamma_{2} \gamma_{3}\right) & \lambda_{100}\left(\left(\gamma_{3}\right)^{2}-\frac{1}{3}\right)
\end{array}\right) \\
& W_{\alpha}^{H}=-\mu_{0} \vec{H} \cdot \vec{M}_{\alpha} \\
& W_{\alpha}^{K}=K_{1}\left(\left(\gamma_{1} \gamma_{2}\right)^{2}+\left(\gamma_{2} \gamma_{3}\right)^{2}+\left(\gamma_{1} \gamma_{3}\right)^{2}\right) \\
& W_{\alpha}^{\sigma}=-\boldsymbol{\sigma}: \epsilon_{\alpha}^{\mu} \\
& \vec{n}_{c}={ }^{t}\left[\cos \left(\phi_{c}\right) \sin \left(\theta_{c}\right), \sin \left(\phi_{c}\right) \sin \left(\theta_{c}\right), \cos \left(\theta_{c}\right)\right] \\
& {\left[\gamma_{1}, \gamma_{2}, \gamma_{3}\right]=\left[\cos \left(\phi_{\alpha}\right) \sin \left(\theta_{\alpha}\right), \sin \left(\phi_{\alpha}\right) \sin \left(\theta_{\alpha}\right), \cos \left(\theta_{\alpha}\right)\right]}
\end{aligned}
$$

The volume fraction $f_{\alpha}$ of a domain is calculated thanks to statistical Boltzmann formula:

$$
f_{\alpha}=\frac{\exp \left(-A_{s} \cdot W_{\alpha}\right)}{\sum_{\alpha} \exp \left(-A_{s} \cdot W_{\alpha}\right)}
$$

$A_{s}$ is an adjusting parameter proportional to the initial susceptibility $\chi_{0}$ of the magnetization curve. We get [8]:

$$
A_{s}=\frac{3 \chi_{0}}{\mu_{0} \cdot M_{s}^{2} \cdot\left(\cos \phi_{c} \sin \theta_{c}\right)^{2}}
$$

The magnetization direction of a domain is defined by angles $\phi_{\alpha}$ and $\theta_{\alpha}$ of the spherical frame (21). The restriction of the loading axis to standard triangle allows to express constitutive laws for the angles of each domain as function of magnetic field, stress, and loading direction parameters (see [8] for more details).

Average magnetization and magnetostriction (24) are projected along the loading axis $\vec{n}_{c}$ leading to the behavior of the single crystal $M(H, \sigma)$ and $\epsilon_{/ /}^{\mu}(H, \sigma)$ (25). Because all possible loading directions are restricted to the standard

triangle, the behavior of an isotropic polycrystal is necessary given by a loading along a single direction inside the triangle. Since behaviors are not linear and single crystal anisotropic, this direction is not the average direction and is theoretically changing with stress or magnetic field level. We consequently make the assumption that this change is small enough to be neglected associated to usual homogeneous field and stress hypotheses over the grains. Parameters to be identified are finally $\phi_{c}$ and $\theta_{c}$ that requires few experimental data (e.g. magnetic and magnetostrictive curves under unstress condition using a least square method ${ }^{2}$ ).

$$
\begin{gathered}
\vec{M}=\sum_{\alpha} f_{\alpha} \vec{M}_{\alpha} \quad \boldsymbol{\epsilon}^{\mu}=\sum_{\alpha} f_{\alpha} \epsilon_{\alpha}^{\mu} \\
M=\vec{M} \cdot \vec{n}_{c} \quad \epsilon_{/ /}^{\mu}={ }^{t} \vec{n}_{c} \cdot \epsilon^{\mu} \cdot \vec{n}_{c}
\end{gathered}
$$

\section{B. Application to the elasto-plastic composite material}

We consider a plastified material composed of hard $(h)$ and soft $(s)$ phases. We suppose on the other hand that macroscopic stress $\boldsymbol{\Sigma}$, kinematic hardening $\mathbf{X}$ and volume fraction of $s$ and $h$ phases are known. The stress field within the two phases is consequently defined $\left(\boldsymbol{\sigma}_{s}, \boldsymbol{\sigma}_{h}\right)$. Considering finally homogeneous magnetic field condition and assuming that the magnetic behavior of each phase is known, a mixing law allows the estimation of the magneto-mechanical behavior of the whole material:

$$
\begin{aligned}
\vec{M}(\vec{H}, \boldsymbol{\Sigma}) & =f_{s} \vec{M}_{s}\left(\vec{H}, \boldsymbol{\sigma}_{s}\right)+f_{h} \vec{M}_{h}\left(\vec{H}, \boldsymbol{\sigma}_{h}\right) \\
\boldsymbol{\epsilon}^{\mu}(\vec{H}, \boldsymbol{\Sigma}) & =f_{s} \boldsymbol{\epsilon}_{s}^{\mu}\left(\vec{H}, \boldsymbol{\sigma}_{s}\right)+f_{h} \boldsymbol{\epsilon}_{h}^{\mu}\left(\vec{H}, \boldsymbol{\sigma}_{h}\right)
\end{aligned}
$$

Magnetization and magnetostriction of the $s$ and $h$ phases can be modeled separately thanks to the multidomain modeling.

A first step is to get the parameters of each phase (loading axis and constant $A_{s}$ ). A second step is to change the multiaxial stresses $\boldsymbol{\sigma}_{s}$ and $\boldsymbol{\sigma}_{h}$ into uniaxial magneto-mechanical equivalent stresses according to the direction of the magnetic loading. We use for that purpose the simplified equivalent stress recently defined in [14]:

$$
\sigma_{i}^{e q}=\frac{3}{2}{ }^{t} \vec{n} \mathbf{S}_{i} \vec{n}
$$

Index $i$ indicates $s$ or $h$ phase. $\mathbf{S}_{i}$ is the deviatoric tensor associated to $\sigma_{s}$ and $\sigma_{h}$ respectively. $\vec{n}$ indicates the direction of the magnetic loading.

\section{APPliCATION TO TENSILE STRENGTHENING}

A tensile loading of axis $\vec{x}$ is considered leading to an axial plastic deformation $E^{p}$. The material can be reloaded along the same direction so that the macroscopic stress tensor is:

$$
\boldsymbol{\Sigma}=\left(\begin{array}{ccc}
\Sigma & 0 & 0 \\
0 & 0 & 0 \\
0 & 0 & 0
\end{array}\right)_{(\vec{x}, \vec{y}, \vec{z})}
$$

\footnotetext{
${ }^{2}$ A separate optimization of $A_{s}$ is possible allowing a better fitting of experimental results.
} 


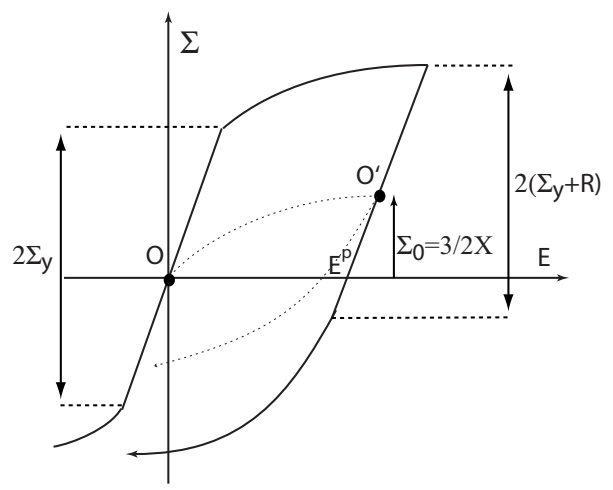

Fig. 3. Kinematic and isotropic variables identification in a classical stressstrain diagram.

The macroscopic plastic strain tensor is constant, diagonal and deviatoric, as well as the kinematic hardening :

$$
\mathbf{E}^{p}=\left(\begin{array}{ccc}
E^{p} & 0 & 0 \\
0 & -\frac{E^{p}}{2} & 0 \\
0 & 0 & -\frac{E^{p}}{2}
\end{array}\right) \mathbf{X}=\left(\begin{array}{ccc}
X & 0 & 0 \\
0 & -\frac{1}{2} X & 0 \\
0 & 0 & -\frac{1}{2} X
\end{array}\right)
$$

The center of the yield surface $O^{\prime}$ is given by $\Sigma_{0}=\frac{3}{2} X$, as illustrated in figure 3.

After the substitution of the value of $\boldsymbol{\Sigma}$ and $\mathbf{X}$ in equations (9), (10), (11) and (14), the stress tensors in soft and hard phases are deduced:

$$
\begin{gathered}
\boldsymbol{\sigma}_{s}=\left(\begin{array}{ccc}
\Sigma+\frac{3}{2} X & 0 & 0 \\
0 & -\frac{3}{4} X & 0 \\
0 & 0 & -\frac{3}{4} X
\end{array}\right) \\
\boldsymbol{\sigma}_{h}=\left(\begin{array}{ccc}
\Sigma-\frac{f_{s}}{f_{h}} \frac{3}{2} X & 0 & 0 \\
0 & \frac{f_{s}}{f_{h}} \frac{3}{4} X & 0 \\
0 & 0 & \frac{f_{s}}{f_{h}} \frac{3}{4} X
\end{array}\right)
\end{gathered}
$$

Considering on the other hand a magnetic loading along $\vec{x}$ axis, the magneto-mechanical equivalent stresses in $s$ and $h$ phases are:

$$
\sigma_{s}^{e q}=\Sigma-\frac{9}{4} X \quad \sigma_{h}^{e q}=\Sigma+\frac{f_{s}}{f_{h}} \frac{9}{4} X
$$

The following points can be highligthed:

- At zero applied stress, we observe that the $s$ phase is submitted to compression, the $h$ phase to traction. This result joins the hypotheses of Cullity [5] in order to interpret the results carried out on a plastic strained ironsilicon alloy (the $h$ phase was actually corresponding to the grain boundaries of the material).

- In order to annul the equivalent stress in the $s$ phase, a tensile stress must be superimposed:

$$
\Sigma=\frac{3}{2} \Sigma_{0}=\frac{9}{4} X
$$

The equivalent stress in the $h$ phase is non zero:

$$
\sigma_{h}^{e q}=\frac{\Sigma_{0}}{f_{h}} \neq 0
$$

This result joins the experimental observations of Iordache [15]. Iordache observed a recovery of the behavior of $\mathrm{Fe}-3 \% \mathrm{Si}$ laminations after plastic deformation for a superimposed stress $\Sigma=\frac{3}{2} \Sigma_{0}$. The $h$ phase corresponding to the grain boundaries of the material does not participate to the magnetic behavior although the stress in the $h$ phase is high. The reason is that the volume fraction of grain boundaries is negligible compared to the volume fraction of matrix.

- In order to annul the equivalent stress in the $h$ phase, a compressive stress must be superimposed:

$$
\Sigma=-\frac{3}{2} \frac{f_{s}}{f_{h}} \Sigma_{0}=-\frac{9}{4} \frac{f_{s}}{f_{h}} X
$$

The equivalent stress in the $s$ phase is non zero:

$$
\sigma_{s}^{e q}=-\frac{\Sigma_{0}}{f_{h}} \neq 0
$$

It could be interesting to propose an experimental validation of such a situation.

\section{CONCLUSION}

In this work a new modeling of the influence of plastic straining on the magnetic and magnetostrictive behaviors of ferromagnetic materials is proposed. This modeling especially dedicated to non destructive evaluation requires to consider the plastified material as a two phased material submitted to residual stresses. The multiaxial stress field within the two phases is associated to the kinematic hardening that can be experimentally measured and depends on the volume fraction of the phases. The magneto-mechanical modeling is made thanks to the so-called multidomain model: the magnetic quantities are calculated separately. An averaging operation leads to the calculation of the macroscopic magnetization and magnetostriction. Considering tensile strengthening, this work gives a theoretical background for the interpretation of previous experimental results of Cullity and Iordache. In [13], an experimental validation of the approach is proposed using a dual-phase steel. Non destructive evaluation remains a far issue since inverse identification procedure must be proposed involving both mechanical and magnetic finite element modeling for an application to non-homogeneous situations.

\section{REFERENCES}

[1] E.Hug, et al., M., Mat. Sc. and Eng., A332, (2002), p.193.

[2] J.M.Makar, B.K. Tanner, J. of Magn. Magn. Mater., 184, (1998) p.193.

[3] J.M.Makar, B.K. Tanner, J. of Magn. Magn. Mater., 187, (1998) p.353.

[4] O. Hubert, et al., Steel Res. Int., 6, (2005), p.440.

[5] B.D. Cullity, Introduction to magnetic Materials, Addison-Wesley Publishing Company, 1972.

[6] O. Hubert, L. Daniel, J. of Magn. Magn .Mater., 304, (2006), p.e489.

[7] M.J. Sablik, et al., IEEE Trans. Mag., 40, (2004), p.3219.

[8] S. Lazreg, O. Hubert, J. Appl. Phys., 109, (2011), 07E508.

[9] H. Mughrabi, Acta Metallurgica, 9 (1983), p.1367.

[10] L. Daniel et al., J. Mech. Phys. Sol., 56 (2008), p.1018.

[11] R. Hill, J. Mech. Phys. Sol., 13 (1965), p.89.

[12] J. Lemaitre, J.L. Chaboche Mechanics of solid materials, Cambridge University Press, 1994.

[13] S. Lazreg, O. Hubert, Influence of plasticity on magnetic and magnetostrictive behaviors of dual-phase steel, SMM20, (2011).

[14] O. Hubert, L. Daniel, J. of Magn. Magn. Mater., 323, (2011), p.1766.

[15] V.E. Iordache, et al., Mater. Sci. Eng. A. 359, (2003) p.62. 